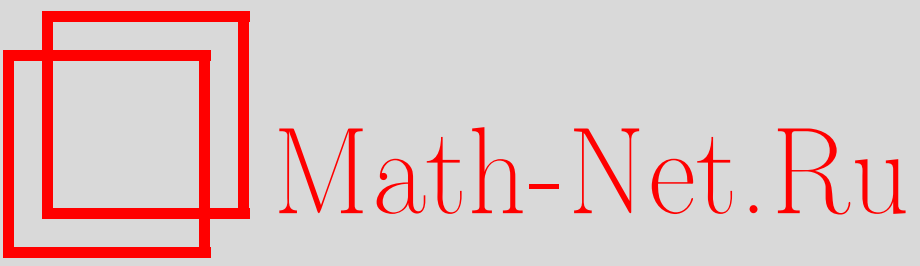

М. В. Шамолин, Топографические системы Пуанкаре и системы сравнения малых и высоких порядков, Итоги науки и техн. Сер. Соврем. мат. и ее прил. Tемат. обз., 2020, том 187, 50-67

DOI: https://doi.org/10.36535/0233-6723-2020-187-50-67

Использование Общероссийского математического портала Math-Net.Ru подразумевает, что вы прочитали и согласны с пользовательским соглашением

http://www.mathnet.ru/rus/agreement

Параметры загрузки:

IP : 107.22 .136 .117

26 апреля 2023 г., 18:09:27 


\title{
ТОПОГРАФИЧЕСКИЕ СИСТЕМЫ ПУАНКАРЕ И СИСТЕМЫ СРАВНЕНИЯ МАЛЫХ И ВЫСОКИХ ПОРЯДКОВ
}

\author{
(c) 2020 г. $\quad$ М. В. ШАМОЛИН
}

\begin{abstract}
АннотАция. В данной работе затрагиваются некоторые качественные вопросы теории обыкновенных дифференциальных уравнений, от решения которых зависит исследование ряда динамических систем. Элементарному обзору подлежат такие проблемы как качественные вопросы теории топографических систем Пуанкаре и более общих систем сравнения; проблемы существования и единственности траекторий, имеющих в качестве предельных множеств бесконечно удаленные точки для систем на плоскости; элементы качественной теории монотонных векторных полей.
\end{abstract}

Ключевые слова: динамическая система, топографическая система Пуанкаре, система сравнения, интегрируемость.

\section{TOPOGRAPHIC POINCARÉ SYSTEMS AND COMPARISON SYSTEMS OF SMALL AND HIGH ORDERS}

\author{
(c) 2020 M. V. SHAMOLIN
}

\begin{abstract}
On this work, we consider some qualitative questions of the theory of ordinary differential equations, on whose solutions a study of a series of dynamical systems depends. An elementary survey is given for such problems as qualitative questions of the theory of topographic Poincaré systems and more general comparison systems; problems of the existence and uniqueness of trajectories having infinitely distant points for flat systems as limit sets; elements of the qualitative theory of monotone vector fields.
\end{abstract}

Keywords and phrases: dynamical system, topographic Poincaré system, comparison system, integrability.

AMS Subject Classification: 34Cxx, 70Cxx

\section{СОДЕРЖАНИЕ}

1. Естественные топографические системы Пуанкаре в системах с диссипацией . . . . . 51

2. Кривые контактов и системы сравнения. Замечания о предельных циклах и проблеме различения центра и фокуса . . . . . . . . . . . . . . . . . . . . . . . . . . . 56

3. О траекториях, имеющих в качестве предельных множеств бесконечно удаленные точки плоскости . . . . . . . . . . . . . . . . . . . . . . . 62

4. Многомерные топографические системы Пуанкаре и системы сравнения . . . . . . . . 65 Список литературы . . . . . . . . . . . . . . . . . . . . . . . . . . 66 


\section{1. ЕСТЕСТВЕННЫЕ ТОПОГРАФИЧЕСКИЕ СИСТЕМЫ ПУАНКАРЕ В СИСТЕМАХ С ДИССИПАЦИЕЙ}

Сначала рассмотрим динамические системы на двумерной плоскости, хотя все сказанное можно перенести и на гладкие ориентированные двумерные многообразия.

Для исследования замкнутых траекторий динамических систем, возникающих в различных приложениях, применим (а в некоторых случаях и видоизменим) теорию топографических систем Пуанкаре (ТСП) $[1,2,14]$.

1.1. Топографические системы Пуанкаре. Пуанкаре предложил метод (хотя и не совсем общий) отыскания замкнутых орбит системы гладких дифференциальных уравнений

$$
\left\{\begin{array}{l}
\dot{x}_{1}=X_{1}\left(x_{1}, x_{2}\right), \\
\dot{x}_{2}=X_{2}\left(x_{1}, x_{2}\right) .
\end{array}\right.
$$

Для этого ему потребовалось ввести понятие «топографических систем» (см. [14]). В его трудах впервые рассматривалась алгебраическая функция $F\left(x_{1}, x_{2}\right)$, обладающая следующими свойствами:

(1) $F\left(x_{1}, x_{2}\right)$ достаточно гладка и ограничена в любой ограниченной области и стремится к бесконечности, когда одна из ее переменных стремится к бесконечности;

(2) $F\left(x_{1}, x_{2}\right)$ равна нулю при $x_{1}=x_{2}=0$ и положительна в остальных точках;

(3) $F\left(x_{1}, x_{2}\right)$ имеет первые производные, обращающиеся в нуль лишь при $x_{1}=x_{2}=0$ (по крайней мере, вблизи начала координат);

(4) $F\left(x_{1}, x_{2}\right)$ такова, что при $x_{1}=x_{2}=0$ выполнено неравенство

$$
\begin{aligned}
& \left(\frac{\partial X_{1}}{\partial x_{2}} \frac{\partial^{2} F}{\partial x_{1}^{2}}-\left(\frac{\partial X_{1}}{\partial x_{1}}-\frac{\partial X_{2}}{\partial x_{2}}\right) \frac{\partial^{2} F}{\partial x_{1} \partial x_{2}}-\frac{\partial X_{2}}{\partial x_{1}} \frac{\partial^{2} F}{\partial x_{2}^{2}}\right)^{2}+ \\
& +4\left(\frac{\partial X_{1}}{\partial x_{1}} \frac{\partial X_{2}}{\partial x_{2}}-\frac{\partial X_{1}}{\partial x_{2}} \frac{\partial X_{2}}{\partial x_{1}}\right)\left(\left(\frac{\partial^{2} F}{\partial x_{1} \partial x_{2}}\right)^{2}-\frac{\partial^{2} F}{\partial x_{1}^{2}} \frac{\partial^{2} F}{\partial x_{2}^{2}}\right)<0
\end{aligned}
$$

которое означает, что кривая контактов имеет в начале координат изолированную особую точку (о кривых контактов см. ниже; см. также $[13,23,24])$;

(5) $F\left(x_{1}, x_{2}\right)$ такова, что кривая, заданная уравнением

$$
X_{1} \frac{\partial F}{\partial x_{1}}+X_{2} \frac{\partial F}{\partial x_{2}}=0
$$

не уходит на бесконечность.

При выполнении условий (1)-(5) уравнение

$$
F\left(x_{1}, x_{2}\right)=\text { const }
$$

дает так называемую топографическую систему вложенных друг в друга кривых, имеющую вершину в начале.

Надо сказать, что не все аналитические условия (1)-(5) нам понадобятся. Мы будем учитывать лишь геометрию расположения кривых контактов, траекторий исследуемой динамической системы и кривых топографической системы Пуанкаре (ТСП).

1.2. Примеры из динамики. 1. Рассмотрим системы вида

$$
\left\{\begin{array}{l}
\dot{\alpha}=-\Omega+A_{1} \delta(\alpha), \\
\dot{\Omega}=F(\alpha), A_{1} \geqslant 0,
\end{array}\right.
$$

где $\delta(\alpha)$ - достаточно гладкая $2 \pi$-периодическая функция, $F$-достаточно гладкая нечетная $\pi$ периодическая функция, удовлетворяющая следующим условиям: $F(\alpha)>0$ при $\alpha \in(0, \pi / 2)$, $d F(0) / d \alpha>0, d F(\pi / 2) / d \alpha<0$ (класс функций $\{F\}=\Phi)$. Таким образом,

$$
F \in \Phi \text {. }
$$


В частности, аналитическая функция

$$
F=F_{0}(\alpha)=\sin \alpha \cos \alpha \in \Phi
$$

также является типичным представителем класса функций $\Phi$ (соответствует так называемому случаю С. А. Чаплыгина в динамике; см. $[17,18])$.

Систему (1.2) при $A_{1}=0$ будем называть системой $\left(1.2^{\prime}\right)$. Для системы $\left(1.2^{\prime}\right)$ начало координат является особой точкой, имеющей топологический тип центра. Таким образом, существует семейство периодических траекторий, подходящих как угодно близко к точкам

$$
\left(-\frac{\pi}{2}, 0\right),\left(\frac{\pi}{2}, 0\right)
$$

В частности, в динамике твердого тела, взаимодействующего с сопротивляющейся средой (см. [11, $12,15])$, система (1.2) принимает более частный вид:

$$
\left\{\begin{array}{l}
\dot{\alpha}=-\Omega+A_{1} \frac{F(\alpha)}{\cos \alpha}, \\
\dot{\Omega}=A_{2} F(\alpha), A_{1}, A_{2} \geqslant 0,
\end{array}\right.
$$

где выполнено свойство (1.3).

2. Рассмотрим системы вида

$$
\begin{aligned}
& \left\{\begin{array}{l}
\alpha^{\prime}=-\omega+b \omega^{2} \delta_{1}(\alpha)+b_{1} F(\alpha) \tilde{f}_{1}(\alpha), \\
\omega^{\prime}=F(\alpha)-\omega \Psi(\alpha, \omega), \quad b, b_{1} \geqslant 0,
\end{array}\right. \\
& \Psi(\alpha, \omega)=-b \omega^{2} \tilde{\delta}_{1}(\alpha)+b_{1} F(\alpha) \delta_{1}(\alpha), \\
& \tilde{\delta}_{1}(\alpha)=\frac{d \delta_{1}(\alpha)}{d \alpha}, \quad \tilde{f}_{1}(\alpha)=\frac{\mu_{1}-\delta_{1}^{2}(\alpha)}{\tilde{\delta}_{1}(\alpha)}, \quad \mu_{1}=\mathrm{const},
\end{aligned}
$$

где $\delta_{1}(\alpha)$ - достаточно гладкая $2 \pi$-периодическая функция, и выполнено свойство (1.3).

Систему (1.7) при $b=b_{1}=0$ будем называть системой $\left(1.7^{\prime}\right)$. Для системы $\left(1.7^{\prime}\right)$ начало координат является особой точкой, имеющей топологический тип центра. Таким образом, существует семейство периодических траекторий, подходящих как угодно близко к точкам (1.5).

В частности, в динамике твердого тела, взаимодействующего с сопротивляющейся средой (см. $[20,22,25])$, система (1.7) принимала более частный вид:

где

$$
\left\{\begin{array}{l}
\alpha^{\prime}=-\omega+\frac{\sigma}{I} F(\alpha) \cos \alpha+\sigma \omega^{2} \sin \alpha, \\
\omega^{\prime}=\frac{1}{I} F(\alpha)-\omega \Psi(\alpha, \omega), \quad \sigma, I>0,
\end{array}\right.
$$

где выполнено свойство (1.3).

$$
\Psi(\alpha, \omega)=\frac{\sigma}{I} F(\alpha) \sin \alpha-\sigma \omega^{2} \cos \alpha,
$$

3. Рассмотрим системы вида

$$
\begin{aligned}
& \left\{\begin{array}{l}
\alpha^{\prime}=-\omega+b \omega^{2} \delta_{2}(\alpha)+b_{1} F(\alpha) \tilde{f}_{2}(\alpha)+b_{2} s(\alpha) \delta_{2}(\alpha), \\
\omega^{\prime}=F(\alpha)-\omega \Psi(\alpha, \omega), b, b_{1}, b_{2} \geqslant 0,
\end{array}\right. \\
& \Psi(\alpha, \omega)=-b \omega^{2} \tilde{\delta}_{2}(\alpha)+b_{1} F(\alpha) \delta_{2}(\alpha)-b_{2} s(\alpha) \tilde{\delta}_{2}(\alpha), \\
& \tilde{\delta}_{2}(\alpha)=\frac{d \delta_{2}(\alpha)}{d \alpha}, \quad \tilde{f}_{2}(\alpha)=\frac{\mu_{2}-\delta_{2}^{2}(\alpha)}{\tilde{\delta}_{2}(\alpha)}, \quad \mu_{2}=\mathrm{const},
\end{aligned}
$$

где $\delta_{2}(\alpha)$ - достаточно гладкая $2 \pi$-периодическая функция, выполнено свойство (1.3), а также выполнено свойство

$$
s \in \Sigma \text {. }
$$

Вводимый класс $\Sigma$ динамических функций достаточно широк: он состоит из функций гладких, $2 \pi$-периодических, четных, удовлетворяющих следующим условиям: $s(\alpha)>0$ при $\alpha \in(0, \pi / 2)$, 
$s(\alpha)<0$ при $\alpha \in(\pi / 2, \pi)$, причем $s(0)>0, d s(\pi / 2) / d \alpha<0$ (класс функций $\{s\}=\Sigma)$. Функция $s$ меняет знак при замене $\alpha$ на $\alpha+\pi$.

В частности, аналитическая функция

$$
s(\alpha)=s_{0}(\alpha)=\cos \alpha \in \Sigma
$$

служит типичным представителем описанного класса и соответствует функции воздействия среды, полученной в одной из своих работ С. А. Чаплыгиным (см. $[17,18,26,27])$ при исследовании плоскопараллельного обтекания плоской пластины бесконечной длины однородным потоком среды.

Систему (1.9) при $b=b_{1}=b_{2}=0$ будем называть системой $\left(1.9^{\prime}\right)$. Для системы $\left(1.9^{\prime}\right)$ начало координат является особой точкой, имеющей топологический тип центра. Таким образом, существует семейство периодических траекторий, подходящих как угодно близко к точкам (1.5).

В частности, в динамике твердого тела, взаимодействующего с сопротивляющейся средой (см. $[32,33,35])$, система (1.9) принимала более частный вид:

$$
\left\{\begin{array}{l}
\alpha^{\prime}=-\omega+\frac{\sigma}{I} F(\alpha) \cos \alpha+\sigma \omega^{2} \sin \alpha+\frac{s(\alpha)}{m} \sin \alpha \\
\omega^{\prime}=\frac{1}{I} F(\alpha)-\omega \Psi(\alpha, \omega), \quad \sigma, I, m>0
\end{array}\right.
$$

где

$$
\Psi(\alpha, \omega)=-\sigma \omega^{2} \cos \alpha+\frac{\sigma}{I} F(\alpha) \sin \alpha-\frac{s(\alpha)}{m} \cos \alpha,
$$

где выполнены свойства (1.3), (1.10).

Изучаемые в примерах системы можно рассматривать или на фазовом цилиндре

$$
\mathbb{S}^{1}\{\alpha \bmod 2 \pi\} \times \mathbb{R}^{1}\{\omega(\text { или } \Omega)\},
$$

или на плоскости $\mathbb{R}^{2}\{\alpha, \omega$ (или $\Omega$ ) $\}$.

Если не будет дополнительно оговорено, можно считать, что выполнены условия (1.3), (1.10).

1.3. Более общее понятие ТСП. Под ТСП будем понимать систему вложенных друг в друга замкнутых кривых, полученных с помощью поверхностей уровня неотрицательной функции, которая равна нулю лишь в точке, к которой сходятся полученные вложенные замкнутые кривые. С помощью такой системы можно успешно «ловить» замкнутые траектории исследуемой динамической системы: вычисляя угол между векторами поля, образующими семейство ТСП, и векторами исследуемого поля динамической системы, можно получить информацию о расположении траекторий исследуемого векторного поля. Чтобы доказать отсутствие замкнутых фазовых характеристик, достаточно выполнения неравенства

$$
\frac{\partial F}{\partial x_{1}} X_{1}+\frac{\partial F}{\partial x_{2}} X_{2} \leqslant 0 \quad(\geqslant 0)
$$

Здесь $F\left(x_{1}, x_{2}\right)=$ const - семейство замкнутых кривых, $X=\left\{X_{1}, X_{2}\right\}$-исходное векторное поле (в координатах $x_{1}, x_{2}$ ). Таким образом, с помощью последнего неравенства можно исследовать качественное поведение траекторий исходной системы. Заметим, что векторное поле обязательно должно быть задано в некоторых координатах.

Выше указывалось, что можно определить ТСП более корректно, но это нам не потребуется. Более того, нас интересуют лишь геометрические свойства взаимного расположения кривых ТСП и фазовых кривых исследуемого поля.

1.4. Характеристические функции и кривые контактов векторных полей. С понятием топографической системы Пуанкаре тесно связано понятие характеристической функции двух полей на плоскости. Последняя функция определяет кососимметрическую форму на плоскости. Если $F\left(x_{1}, x_{2}\right)=$ const - семейство замкнутых кривых, то система, имеющая явный вид гамильтоновой,

$$
\dot{x_{1}}=-\frac{\partial F}{\partial x_{2}}, \quad \dot{x_{2}}=\frac{\partial F}{\partial x_{1}}
$$


задает векторное поле, касательное к семейству кривых ТСП. Тогда последнее неравенство эквивалентно неравенству

$$
X_{1} Y_{2}-X_{2} Y_{1} \leqslant 0 \quad(\geqslant 0)
$$

в котором

$$
Y_{1}=-\frac{\partial F}{\partial x_{2}}, \quad Y_{2}=\frac{\partial F}{\partial x_{1}}
$$

- векторное поле системы. Оно касается кривых ТСП.

Рассмотрим две системы уравнений на плоскости. Эти уравнения задаются гладкими векторными полями $X=\left\{X_{1}, X_{2}\right\}$ и $Y=\left\{Y_{1}, Y_{2}\right\}$ в некоторых координатах $x=\left(x_{1}, x_{2}\right)$. Естественно рассмотреть функцию

$$
\chi=\chi(X, Y)=X_{1} Y_{2}-X_{2} Y_{1},
$$

которая отвечает за знак синуса угла между полями $X$ и $Y$. Очевидно, $\chi(X, Y)=0$ там и только там, где поля $X$ и $Y$ касаются.

Функция $\chi$ удовлетворяет следующим свойствам:

$$
\chi(X, Y)=-\chi(Y, X), \quad \chi(\lambda X, Y)=\lambda \chi(X, Y)
$$

для любой действительной функции $\lambda$.

Определение 1.1. Функцию $\chi$ мы назовем характеристической функиией двух векторных полей, а уравнение $\chi(X, Y)=0$ - уравнением кривой контактов для полей $X$ и $Y$.

Введем обозначения полос:

$$
\Pi=\left\{(\alpha, \Omega) \in \mathbb{R}^{2}:-\frac{\pi}{2}<\alpha<\frac{\pi}{2}\right\}, \quad \Pi^{\prime}=\left\{(\alpha, \Omega) \in \mathbb{R}^{2}: \frac{\pi}{2}<\alpha<\frac{3 \pi}{2}\right\} .
$$

В применение метода ТСП к динамическим системам, возникающим в динамике твердого тела, взаимодействующего с сопротивляющейся средой, рассмотрим системы вида (1.2) в полосе П. Ранее (см. $[21,34,36])$ было показано, что при некоторых условиях у данной системы не существует так называемых монотонных предельных циклов. Оказывается, при тех же условиях у такой системы не существует никакой замкнутой кривой, состоящей из фазовых траекторий.

Лемма 1.1. Пусть $A_{1} \neq 0$. Если выполнено неравенство $F(\alpha) \delta(\alpha)>0(<0)$ почти всюду в полосе П, то у системы вида (1.2) не существует замкнутой кривой из траекторий в полосе П.

Доказательство. Систему (1.2) при $A_{1}=0$ будем называть системой $\left(1.2^{\prime}\right)$. Как уже отмечалось, для системы $\left(1.2^{\prime}\right)$ начало координат является особой точкой, имеющей топологический тип центра. Таким образом, существует семейство периодических траекторий, подходящих как угодно близко к точкам $(-\pi / 2,0),(\pi / 2,0)$. Характеристическая функция, отвечающая системам $(1.2)$ и $\left(1.2^{\prime}\right)$, равна $A_{1} F(\alpha) \delta(\alpha)$. Кривая контактов - это объединение конечного числа прямых вида

$$
\left\{(\alpha, \Omega) \in \mathbb{R}^{2}: \alpha=\alpha^{0}\right\}, \quad-\frac{\pi}{2}<\alpha^{0}<\frac{\pi}{2} .
$$

В остальных же точках полосы П характеристическая функция положительна.

Рассуждаем от противного. Предположим, что существует замкнутая кривая из траекторий $\gamma_{0}$, ограничивающая область $S_{0}$. Пусть точка $\left(\alpha_{0}, 0\right) \in \gamma_{0}$, где $0<\alpha_{0}<\pi / 2$. Рассмотрим точку $\left(\alpha_{0}+\varepsilon, 0\right)$, где $\varepsilon$ - достаточно мало. Через нее проходит замкнутая траектория $\gamma_{\varepsilon}$ из ТСП, ограничивающая область $S_{\varepsilon}$. Очевидно, что, выходя из точки $\left(\alpha_{0}, 0\right)$, покидая область $S_{\varepsilon}$, траектория $\gamma_{0}$ никогда не попадет в область $S_{\varepsilon}$, в силу положительности почти всюду характеристической функции. Но $\left(\alpha_{0}, 0\right) \in S_{\varepsilon}$. Противоречие. Лемма доказана.

Следствие 1.1. Если $F \in \Phi, \delta \in Y$, то в полосе $\Pi$ (впрочем, как и в полосе $\Pi^{\prime}$ ) не существует замкнутой характеристики системы (1.2). 
Вводимый класс $Y$ динамических функций достаточно широк: он состоит из функций достаточно гладких, $2 \pi$-периодических, нечетных, удовлетворяющих следующим условиям: $\delta(\alpha)>0$ при $\alpha \in(0, \pi)$, причем $d \delta(0) / d \alpha>0, d \delta(\pi) / d \alpha<0$ (класс функций $\{\delta\}=Y$ ). Функция $\delta$ меняет знак при замене $\alpha$ на $\alpha+\pi$. Таким образом, $\delta \in Y$. В частности, аналитическая функция $\delta(\alpha)=\delta_{0}(\alpha)=\sin \alpha \in Y$ служит типичным представителем описанного класса.

Рассмотрим далее систему (1.7) в полосе П. Аналогично лемме 1.1 доказывается следующее утверждение.

Лемма 1.2. Если выполнены неравенства $\tilde{\delta}_{1}(\alpha), \tilde{f}_{1}(\alpha)>0(<0)$ почти всюду в полосе П, то у системы вида (1.7) в полосе П при $b=b_{1}>0$ не существует замкнутой кривой из траекторий.

Доказательство. Систему (1.7) при $b=b_{1}=0$ будем называть системой $\left(1.7^{\prime}\right)$. Аналогично лемме 1.1, система $\left(1.7^{\prime}\right)$ обладает особой точкой типа центр, окруженной замкнутыми траекториями, продолжающимися до точек $(-\pi / 2,0),(\pi / 2,0)$. Характеристическая функция, отвечающая системам $(1.7)$ и $\left(1.7^{\prime}\right)$, при $b=b_{1}$ равна

$$
b\left\{F^{2}(\alpha) \tilde{f}_{1}(\alpha)+\omega^{4} \tilde{\delta}_{1}(\alpha)\right\} .
$$

Лишь на множестве нулевой меры данная функция обращается в нуль, а в остальных точках она знакопостоянна. Из рассуждений, используемых при доказательстве леммы 1.1, следует леммa 1.2.

Следствие 1.2. Если $F \in \Phi, \tilde{\delta}_{1}, \tilde{f}_{1} \in \Sigma$, то в полосе П (впрочем, как и в полосе П') не существует замкнутой характеристики системы (1.7).

Теперь рассмотрим систему (1.9) в полосе П. Аналогично леммам 1.1, 1.2 доказывается следующая лемма.

Лемма 1.3. Если выполнены неравенства $\tilde{\delta}_{2}(\alpha), \tilde{f}_{2}(\alpha), F(\alpha) \delta_{2}(\alpha)>0(<0)$ почти всюду в полосе П, то у системь вида (1.9) в полосе П при $b=b_{1}, b_{2}>0$ не существует замкнутой кривой из траекторий.

Доказательство. Рассмотрим систему $\left(1.7^{\prime}\right)$ (см. лемму 1.2. Для нее по-прежнему начало координат имеет топологический тип центра, обладающий семейством замкнутых траекторий, продолжающихся до точек $(-\pi / 2,0),(\pi / 2,0)$. Характеристическая функция, отвечающая системам $(1.9)$ и $\left(1.7^{\prime}\right)$, при $b=b_{1}$ равна

$$
b\left\{F^{2}(\alpha) \tilde{f}_{2}(\alpha)+\omega^{4} \tilde{\delta}_{2}(\alpha)\right\}+b_{2}\left\{F(\alpha) s(\alpha) \delta_{2}(\alpha)+\omega^{2} s(\alpha) \tilde{\delta}_{2}(\alpha)\right\} .
$$

Очевидно, что почти всюду в полосе П характеристическая функция положительна. Остается сослаться на методы доказательств лемм 1.1 и 1.2 .

Следствие 1.3. Если $F \in \Phi, s, \tilde{\delta}_{2}, \tilde{f}_{2}, \in \Sigma, \delta_{2} \in Y$, то в полосе П не существует замкнутой характеристики системы (1.9).

Замечание 1.1. Как было показано ранее (см. $[21,37,38])$, в полосе П' у системы вида (1.9) возможны при некоторых условиях простые и сложные предельные циклы.

Рассмотрим систему (1.9) в области

Если

$$
O^{\prime}=\left\{\Pi_{(0, \pi)} \cap\left\{(\alpha, \omega) \in \mathbb{R}^{2}: \omega>0\right\}\right\} .
$$

$$
s\left(\frac{\pi}{2}\right)=F\left(\frac{\pi}{2}\right)=\tilde{\delta}_{2}\left(\frac{\pi}{2}\right)=0, \quad \delta_{2}\left(\frac{\pi}{2}\right) \neq 0,
$$

то при $b>0$ существует особая точка

$$
\left(\frac{\pi}{2}, \frac{1}{b \delta_{2}(\pi / 2)}\right)
$$


Здесь

$$
\Pi_{(0, \pi)}=\left\{(\alpha, \omega) \in \mathbb{R}^{2}: \quad 0<\alpha<\pi\right\} .
$$

Аналогично леммам 1.1-1.3 доказывается следующее утверждение.

Лемма 1.4. Рассмотрим систему вида (1.9) при $b, b_{1}, b_{2}>0$ в области $O^{\prime}$. Пусть для простоты $F \in \Phi, s, \tilde{\delta}_{2}, \tilde{f}_{2} \in \Sigma, \delta_{2} \in Y$. Тогда вокруг точки покоя (1.13) в области $O^{\prime}$ не существует замкнутой кривой из траекторий, если неравенство

$$
\omega^{2} \frac{\tilde{\delta}_{2}(\alpha)}{s(\alpha)}-b_{1} \mu_{2} \frac{F(\alpha)}{s(\alpha)} \omega+\frac{F(\alpha)}{s(\alpha)} \delta_{2}(\alpha)>0
$$

не выполнено в области $O^{\prime}$ только при $\alpha=\pi / 2$.

Заметим, что равенство (1.14) возникает при изучении так называемых нетривиальных положений равновесия (НПР) системы (1.9) (см. [39, 40,42]).

Доказательство. В области $O^{\prime}$ рассмотрим систему (1.9). Нетрудно показать, что, поскольку $F \in \Phi, \tilde{\delta}_{2}, \tilde{f}_{2} \in \Sigma, \delta_{2} \in Y$, то точка покоя (1.13) для системы (1.7) имеет топологический тип центра (см. [43,44]). Существует семейство замкнутых траекторий, окружающее точку (1.13). Это семейство может уходить на бесконечность. Характеристическая функция, отвечающая системам (1.7) и (1.9), равна

$$
b_{2} s^{2}(\alpha)\left\{\omega^{2} \frac{\tilde{\delta}_{2}(\alpha)}{s(\alpha)}-b_{1} \mu_{2} \frac{F(\alpha)}{s(\alpha)} \omega+\frac{F(\alpha)}{s(\alpha)} \delta_{2}(\alpha)\right\} .
$$

Последняя величина знакоопределена, поскольку неравенство (1.14) не выполнено лишь при $\alpha=$ $\pi / 2$. Поля систем (1.7) и (1.9) в области $O^{\prime}$ касаются лишь на прямой $\left\{(\alpha, \omega) \in \mathbb{R}^{2}: \alpha=\pi / 2\right\}$. При этом необходимо заметить, что коэффициенты квадратного относительно $\omega$ неравенства (1.14) обладают следующими свойствами:

$$
\frac{F}{s} \in Y, \quad \frac{\tilde{\delta}_{2}}{s}>0
$$

Остается сослаться на методы доказательств лемм 1.1-1.3.

Вообще говоря, при исследовании системы (1.9), в зависимости от области, используется одна из систем вида $(1.7)$ или $\left(1.7^{\prime}\right)$.

\section{2. КРИВЫЕ КОНТАКТОВ И СИСТЕМЫ СРАВНЕНИЯ.}

ЗАМЕЧАНИЯ О ПРЕДЕЛЬНЫХ ЦИКЛАХ И ПРОБЛЕМЕ РАЗЛИЧЕНИЯ ЦЕНТРА И ФОКУСА

2.1. Системы сравнения и исследование топологической структуры расположения траекторий. Метод ТСП, о котором говорилось в $[23,29]$, является частным случаем метода исследования с помощью систем сравнения.

Рассмотрим две системы уравнений на плоскости и характеристическую функцию определяющих их векторных полей, которая, как указывалось, отвечает за знак синуса угла между векторными полями данных систем. Зная принцип разбиения на траектории одной из них, возможен анализ устройства фазовой плоскости другой системы. В частности, ТСП позволяет, к примеру, исследовать вопрос существования предельных циклов (см. $[3,4,7,8]$ ). Таким образом, основной упор делается на вычисление угла между двумя полями рассматриваемых систем в одной и той же области фазовой поверхности.

Вычислять характеристическую функцию можно для любых двух векторных полей на плоскости. В этой связи назовем системой сравнения для данной системы ту систему, качественное расположение траекторий которой полностью известно.

Ранее уже проводилось сравнение векторных полей систем $\left(1.2^{\prime}\right),(1.9),(1.7)$.

Предложение 2.1. Рассмотрим системы вида (1.7) и (1.9) на плоскости. Система (1.7) является системой сравнения для системы (1.9) следующим образом: почти всюду угол от вектора одного поля до вектора другого поля лежит в пределах от 0 до $\pi$ (с учетом направления), и лишь на множестве нулевой меры этот угол равен нулю, если выполнены все условия леммы 1.4 . 
Доказательство. Действительно, если $F \in \Phi, s \in \Sigma$, то, в силу леммы 1.4, характеристическая функция во всей фазовой плоскости знакоопределена.

Замечание 2.1. Если выполнены условия (1.4), (1.11), то угол между векторами полей систем (1.12) и (1.8) меняется монотонно относительно параметра $\mu_{1}=2 B / m n_{0}$, где

$$
n_{0}^{2}=\frac{d F(0)}{I d \alpha}, \quad B=s(0) .
$$

$\mathrm{B}$ частности, при $\mu_{1}=0$ последняя характеристическая функция тождественно равна нулю.

Замечание 2.2. Поскольку система (1.8) на плоскости имеет три топологически различных типа фазового портрета (см. [37]), для исследования системы (1.12) в каждой из областей ее параметров можно использовать свой топологический тип (см. $[5,10])$.

В качестве системы сравнения для системы (1.9) можно также использовать и систему $\left(1.7^{\prime}\right)$, описывающую консервативную систему, - физический маятник на плоскости.

Замечание 2.3. У системы (1.9) не существует замкнутых кривых из траекторий, огибающих фазовый цилиндр, если выполнены все условия предложения 2.1.

Доказательство. От противного. Пусть существует искомая замкнутая кривая. Можно считать, что начальными условиями для такой траектории является точка $\left(0, \omega^{*}\right), \omega^{*}>0$. Проведем через данную точку траекторию системы сравнения (1.7) в зависимости от топологического типа ее фазового портрета $[28,31]$. В силу предложения 2.1 , если такая траектория существует, то она пройдет лишь через точку $\left(2 \pi, \omega^{*}+\delta\right)$, где $\delta>0$, что противоречит замкнутости кривой.

Следующее предложение сформулируем для наглядности для систем частного вида (1.12). Для систем более общего вида (1.9) данное предложение несложно переписывается.

Предложение 2.2. Рассмотрим системы (1.12) и (1.8') во всей плоскости. В данном случае система (1.8') - это система (1.8) при $\sigma=0$. Система $\left(1.8^{\prime}\right)$ является системой сравнения для системы (1.12) следуюшим образом: в полосе П почти всюду угол от вектора одного поля до вектора другого поля лежит в пределах от 0 до $\pi$ (с учетом направления), и лишь на множестве нулевой меры этот угол равен нулю, а в полосе $\Pi^{\prime}$ при условии, что

$$
\frac{s(\alpha)}{m \cos \alpha} \geqslant \frac{\sigma}{I} \frac{F(\alpha)}{\sin \alpha} \quad \forall \alpha \in\left(0, \frac{\pi}{2}\right),
$$

существует кривая контактов рассматриваемых двух полей, которая является топологической окружностъю.

Доказательство. Действительно, если $F \in \Phi, s \in \Sigma$, то в полосе П кривая контактов - начало координат. Перенесем начало координат в точку $(\pi, 0)$ и перепишем уравнение кривой контактов в виде

$$
\sigma \cos \alpha\left\{\left(\frac{F(\alpha)}{I}\right)^{2}+\omega^{4}\right\}=\frac{1}{I} F(\alpha) \frac{s(\alpha)}{m} \sin \alpha+\omega^{2} \frac{s(\alpha)}{m} \cos \alpha .
$$

Тогда уравнение (2.1) кривой контактов, которую назовем нетривиальной (HKK), можно разрешить относительно $\omega^{2}$ :

$$
2 \sigma \omega^{2}=\frac{s(\alpha)}{m} \pm \sqrt{\frac{s^{2}(\alpha)}{m^{2}}+4 \sigma \frac{F(\alpha)}{I} \sin \alpha\left\{\frac{s(\alpha)}{m \cos \alpha}-\frac{\sigma}{I} \frac{F(\alpha)}{\sin \alpha}\right\}} .
$$

В полосе П уравнение (2.2), взятое со знаком «минус», задает лишь точку $(0,0)$, а взятое со знаком «плюс» - точку $(0,0)$ и НKK.

HKK симметрична относительно обеих осей координат (после переноса из полосы П' в полосу П), пересекает обе оси под прямым углом и только два раза (по теореме о неявной функции). Предложение доказано. 
2.2. Некоторые общие утверждения о ТСП и системах сравнения. С целью рассмотрения вопросов существования ключевых траекторий (замкнутых фазовых характеристик и др.), докажем одну общую теорему. Для этого заметим, что вплоть до прямых $\Lambda_{-1}$ и $\Lambda_{0}$ (а также $\Lambda_{0}$ и $\Lambda_{1}$ ) существует семейство замкнутых кривых, которое является ТСП (интегральные кривые системы $\left(1.8^{\prime}\right)$, которая описывает физический маятник). Здесь

$$
\Lambda_{k}=\left\{(\alpha, \omega) \in \mathbb{R}^{2}: \alpha=\frac{\pi}{2}+\pi k\right\} .
$$

Поставим также вопрос о существовании замкнутых кривых из траекторий для системы (1.12) в полосе П'. Для этого докажем утверждение, обобщающее леммы 1.1-1.4.

Теорема 2.1. Пусть в односвязной области $D$ плоскости, содержащей точку покоя $x_{0}$ достаточно гладкого векторного поля $v_{1}$, существует такая кривая $\gamma \ni x_{0}$, соединяющая две точки $A, B \in \partial D$ (точки $A, B$ могут быть бесконечно удаленъ), что существует ТСП с иен-

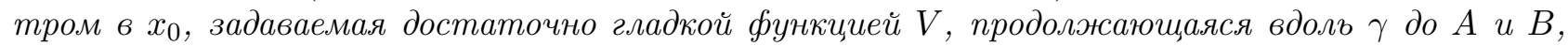
заполняющая область $\mathrm{K} \subseteq D$ и обладающая свойством

$$
\left.\left(v_{1}, v_{2}\right)\right|_{\mathbb{R}^{2}}>0
$$

(где $\left.v_{2}=\operatorname{grad} V\right)$ почти всюду в $\mathrm{K}$, за исключением, быть может, некоторых кривых, не охватывающих $x_{0}$. (Здесь $V=$ const - семейство кривых ТСП.) Тогда во всей области D вокруг точки $x_{0}$ не существует ни одной замкнутой кривой из траекторий поля $v_{1}$.

Доказательство. Предположим, что такая кривая $\gamma_{0}$ существует и ограничивает область $S_{0} \ni x_{0}$. Пусть $\left\{N_{1}, N_{2}\right\}=\gamma \cap \gamma_{0} \neq \emptyset$ (поскольку $x_{0} \in \gamma$ ) и точка $N_{1}$ - неособое начальное условие при движении по кривой $\gamma_{0}$. Через точку $N_{1}$ проходит замкнутая кривая $\bar{\gamma}$ из ТСП, причем $\bar{\gamma} \subset \mathrm{K}$. Если кривая $\bar{\gamma}$ ограничивает область $\bar{S}$, то такое существует $\varepsilon>0$ (которое уменьшим насколько нужно), что имеет место следующее:

(1) $N_{\varepsilon} \in \bar{\gamma}_{\varepsilon} \cap \gamma$, где $\bar{\gamma}_{\varepsilon}-$ кривая ТСП;

(2) расстояние между точками $N_{\varepsilon}$ и $N_{1}$ равно $\varepsilon$;

(3) $\bar{\gamma}_{\varepsilon}$ ограничивает область $\bar{S}_{\varepsilon} \supset \bar{S}$.

Выбранное значение $\varepsilon$ таково, что точка, двигаясь по траектории $\gamma_{0}$ с начальным условием $N_{1}$, покинет область $\bar{S}_{\varepsilon}$ через конечное время. Поскольку $\bar{S}_{\varepsilon} \subset \mathrm{K}$ и неравенство (2.3) выполнено почти всюду в $\mathrm{K}$, за исключением, быть может, некоторых кривых, не охватывающих $x_{0}$, то точка с начальным условием $N_{1}$ никогда больше в область $\bar{S}_{\varepsilon} \subset \mathrm{K} \subset D$ не вернется. Так как $\bar{S} \subset \bar{S}_{\varepsilon}$, то приходим к противоречию с замкнутостью кривой $\gamma_{0}$.

Как уже отмечалось, в полосе П' для систем вида (1.12) при некоторых условиях существует ТСП с центром в точке $(\pi, 0)$ (система $\left(1.8^{\prime}\right)$ ), продолжающаяся до точек

$$
\left(\frac{\pi}{2}, 0\right), \quad\left(\frac{3 \pi}{2}, 0\right) \text {. }
$$

При этом НКК системы сравнения (ТСП) и поля системы (1.12) ограничивает область, целиком содержащую ТСП. Таким образом, ссылаясь на теорему 2.1 , получаем следующее утверждение.

Лемма 2.1. Рассмотрим систему (1.12) в полосе $\Pi^{\prime}$, если НКК системь сравнения (1.8') и системь (1.12) ограничивает область, иеликом содержащую ТСП, продолжающуюся до точек (2.4). Тогда в полосе П' не существует замкнутой кривой из траекторий системы (1.12).

Рассмотрим системы вида (1.12) при условиях (1.3), (1.10). Выше ставился вопрос о существовании замкнутых кривых из траекторий в полосе $\Pi^{\prime}$, т.е. кривых, окружающих точку $(\pi, 0)$. Поставим также более общий вопрос о существовании любых замкнутых кривых из траекторий системы (1.12), стягиваемых по фазовому цилиндру в точку.

Очевидно, что сумма индексов особых точек, находящихся внутри таких кривых, должна равняться 1. Значит такие кривые могут возникнуть вокруг точек $(\pi k, 0), k \in \mathbb{Z}$. Внутри таких 
кривых не могут содержаться одновременно два седла

$$
\left(-\frac{\pi}{2}+2 \pi k, 0\right), \quad\left(\frac{\pi}{2}+2 \pi k, 0\right)
$$

и точка $(2 \pi k, 0)$, поскольку сумма индексов при этом равна -1 . Такие кривые не могут содержать внутри себя одновременно точки $(\pi k, 0)$ и $(\pi(k+1), 0)$, поскольку, в силу центральной симметрии поля системы (1.12) относительно точек $(\pi k, 0)$ и теоремы единственности, это невозможно.

Остается единственная возможность существования такой кривой, содержащей более одной особой точки внутри себя. Пусть для определенности $k=0$. Такая кривая может содержать точки

$$
(0,0), \quad\left(-\frac{\pi}{2}, 0\right), \quad\left(\frac{\pi}{2}, 0\right), \quad\left(-\frac{\pi}{2},-\frac{1}{\sigma}\right), \quad\left(\frac{\pi}{2}, \frac{1}{\sigma}\right),
$$

сумма индексов которых равна 1. Но в силу леммы 1.4 это невозможно, хотя бы потому, что траектория, имеющая в качестве $\omega$-предельного множества точку $(\pi / 2,1 / \sigma)$, имеет в качестве $\alpha$ предельного множества бесконечно удаленную точку (см. [19, 30]).

Таким образом, вопрос существования замкнутых кривых из траекторий системы (1.12), стягиваемых по фазовому цилиндру в точку, при условии выполнения леммы 1.4, свелся к отысканию таких кривых в полосе $\Pi^{\prime}$ вокруг точки $(\pi, 0)$. Как было показано в [37], при некоторых условиях такие кривые существуют.

2.3. Проблема различения центра и фокуса и системы сравнения. Первая проблема различения в качественной теории обыкновенных дифференциальных уравнений - проблема центра и фокуса - возникает в точке $(\pi, 0)$ в полосе $\Pi^{\prime}$ для динамической системы вида (1.12) при условиях (1.3), (1.10), а также при $\mu_{1}=\mu_{2}$ и $0<\mu_{2}<2$ (см. [37]). В [26, 27] данная проблема решена при In $\neq 0$ в пользу слабого фокуса. Таким образом, в достаточно малой окрестности точки $(\pi, 0)$ все траектории-спирали приближаются к точке $(\pi, 0)$ либо при $t \rightarrow+\infty$, либо при $t \rightarrow-\infty$ (здесь $t-$ независимый параметр вдоль траекторий).

Поставим вопрос о расширении данной окрестности точки $(\pi, 0)$ в полосе П' (на плоскости $\left.\mathbb{R}^{2}\{\alpha, \omega\}\right)$. Для этого переведем начало координат в точку $(0,0)$. После такой замены переменных система (1.12) (рассматриваемая уже в полосе П) примет вид

$$
\left\{\begin{array}{l}
\alpha^{\prime}=-\omega-\frac{\sigma}{I} F(\alpha) \cos \alpha-\sigma \omega^{2} \sin \alpha+\frac{s(\alpha)}{m} \sin \alpha \\
\omega^{\prime}=\frac{1}{I} F(\alpha)-\sigma \omega^{3} \cos \alpha+\frac{\sigma}{I} \omega F(\alpha) \sin \alpha+\frac{\omega}{m} s(\alpha) \cos \alpha .
\end{array}\right.
$$

Для простоты рассмотрим систему (2.5) при условиях (1.4), (1.11), т.е. когда $F(\alpha)=F_{0}(\alpha)$, $s(\alpha)=s_{0}(\alpha)$. После замены в полосе $\Pi$ по формуле $\tau=\sin \alpha,|\tau|<1$, системе $(2.5)$ сопоставим уравнение

$$
\frac{d \omega}{d \tau}=\frac{n_{0}^{2} \tau-\sigma \omega^{3}+\sigma n_{0}^{2} \omega \tau^{2}+\frac{\sigma n_{0}^{2}}{2} \omega \sqrt{1-\tau^{2}}}{-\omega-\sigma n_{0}^{2} \tau\left(1-\tau^{2}\right)-\sigma \omega^{2} \tau+\frac{\sigma n_{0}}{2} \tau \sqrt{1-\tau^{2}}}
$$

В уравнении (2.6) уже учтено условие $\mu_{1}=\mu_{2}$, которое эквивалентно

$$
\frac{B}{m n_{0}}=\frac{\sigma n_{0}}{2} \text {. }
$$

Пусть формально $\tau=x, \omega=-y$. Фазовые траектории системы $(2.5)$, рассматриваемой в полосе П, совпадают с интегральными траекториями уравнения (2.6), которое, в свою очередь, рассматривается в полосе $\left\{(x, y) \in \mathbb{R}^{2}:-1<x<1\right\}$. Система приводится к виду

$$
\left\{\begin{array}{l}
x^{\prime}=y-\sigma n_{0}^{2} x+\sigma n_{0}^{2} x^{3}-\sigma y^{2} x+\frac{\sigma n_{0}}{2} x \sqrt{1-x^{2}}, \\
y^{\prime}=-n_{0}^{2} x-\sigma y^{3}+\sigma n_{0}^{2} y x^{2}+\frac{\sigma n_{0}^{2}}{2} y \sqrt{1-x^{2}}
\end{array}\right.
$$


Рассмотрим систему

$$
\left\{\begin{array}{l}
x^{\prime}=y-\frac{\sigma n_{0}^{2}}{2} x+\frac{3 \sigma n_{0}^{2}}{4} x^{3}-\sigma y^{2} x, \\
y^{\prime}=-n_{0}^{2} x+\frac{\sigma n_{0}^{2}}{2} y-\sigma y^{3}+\frac{3 \sigma n_{0}^{2}}{4} y x^{2},
\end{array}\right.
$$

которая является системой сравнения для системы (2.7). Характеристическая функция пары систем (2.7) и (2.8) является, как уже отмечалось, кососимметрической билинейной функцией.

Для упорядоченной пары систем $X$ и $Y$ их характеристическую функцию будем обозначать через $\chi(X, Y)$.

Предложение 2.3. Характеристическая функиия $\chi((2.7),(2.8))$ при бn $<2$ в полосе $\left\{(x, y) \in \mathbb{R}^{2}:-1<x<1\right\}$ положительно определена (она равна нулю лишь в начале координат).

Доказательство. Действительно,

$$
\chi((2.7),(2.8))=\frac{\sigma n_{0}^{2}}{2}\left[y^{2}-\sigma n_{0}^{2} x y+n_{0}^{2} x^{2}\right]\left[1-\frac{x^{2}}{2}-\sqrt{1-x^{2}}\right] .
$$

Заметим, что правые части системы (2.8) отличаются от правых частей системы (2.7) лишь членами пятого порядка малости по $\rho=\left(x^{2}+y^{2}\right)^{1 / 2}$, т.е. членами порядка $O\left(\rho^{5}\right)$. В связи со сделанным только что замечанием о представлении векторного поля системы (2.7) около точки $(0,0)$, справедливо следующее предложение.

Предложение 2.4. Точка $(0,0)$ является сложным устойчивым фокусом при $\sigma n_{0}<2$ для системы (2.8).

Доказательство. Действительно, индекс In (см. [2,37]) точки $(0,0)$ для системы (2.8) совпадает с индексом In точки $(0,0)$ для системы $(2.7)$ и он отрицателен, поскольку зависит лишь от вторых и третьих производных правых частей рассматриваемых систем.

Поставим следующий вопрос: как соотносится знак характеристической функции как формы от упорядоченной пары систем на плоскости и угол поворота от поля одной системы к другой. Для этого рассмотрим систему

$$
\left\{\begin{array}{l}
x^{\prime}=y-\sigma n_{0}^{2} x+\sigma n_{0}^{2} x^{3}-\sigma y^{2} x+\lambda x \sqrt{1-x^{2}}, \\
y^{\prime}=-n_{0}^{2} x-\sigma y^{3}+\sigma n_{0}^{2} y x^{2}+\lambda y \sqrt{1-x^{2}},
\end{array}\right.
$$

зависящую от параметра $\lambda>0$. Векторное поле системы (2.9) обладает некоторым свойством строгой монотонности (CM) (см. далее) при некоторых условиях и при $\sigma n_{0}<2$ в любой области без особых точек. Если при $\lambda=\lambda_{1}$ систему (2.9) обозначить через $\left(2.9^{\prime}\right)$, а при $\lambda=\lambda_{2}-$ через $\left(2.9^{\prime \prime}\right)$, то справедливо равенство

$$
\chi\left(\left(2.9^{\prime}\right),\left(2.9^{\prime \prime}\right)\right)=\left(\lambda_{2}-\lambda_{1}\right)\left[y^{2}-\sigma n_{0}^{2} x y+n_{0}^{2} x^{2}\right] \sqrt{1-x^{2}} .
$$

Таким образом, при $\lambda_{2}>\lambda_{1}$ последняя функция положительно определена в полосе $\left\{(x, y) \in \mathbb{R}^{2}\right.$ : $-1<x<1\}$ (равна нулю лишь в начале координат). Но как легко понять, начало координат для системы $\left(2.9^{\prime \prime}\right)$ является «более неустойчивой» особой точкой, чем начало координат для системы $\left(2.9^{\prime}\right)$. Таким образом, векторное поле системы $\left.(2.9)^{\prime \prime}\right)$ поворачивается при $\lambda_{2}>\lambda_{1}$ около векторного поля системы $\left(2.9^{\prime}\right)$ на положительный угол.

Прежде чем говорить о характере траекторий системы (2.7), сформулируем одно вспомогательное утверждение, представляющее самостоятельный интерес.

Для поиска подходящей системы сравнения, в целях исследования существования предельных циклов, проблемы различения центра и фокуса и т.д., вовсе не обязательно иметь ТСП с центром в данной особой точке. Искомая система сравнения может иметь либо притягивающую, либо отталкивающую особую точку. 
Пусть в области $D$, содержащей единственную особую точку системы $(\mathrm{A})$, заданной для простоты на плоскости, стоит проблема различения центра и фокуса. Пусть в этой же области система (Б) имеет ту же единственную особую точку $x_{0}$.

Рассмотрим для определенности так называемые отрицательно ориентируемые системы, в которых траектории обходят точку $x_{0}$ против часовой стрелки. Аналогично могут быть рассмотрены положительно ориентируемые системы.

Лемма 2.2. Пусть область $D$ является притягиваемой (отталкиваемой) точкой $x_{0}$ отрицательно ориентируемой системы (Б). Тогда если характеристическая функиия ұ((А),(Б)) положительно (отрицательно) определена в области $D$, то область $D$ является притягиваемой (отталкиваемой) точкой $x_{0}$ (отрицательно ориентируемой) системъ (A).

Лемма 2.2 носит явно геометрический характер. Действительно, векторное поле системы (Б) повернуто относительно векторного поля системы $(\mathrm{A})$ на неотрицательный (неположительный) угол.

Аналогичное утверждение можно сформулировать и для устойчивых и неустойчивых предельных циклов и т.д.

Итак, в качестве системы (А) возьмем систему (2.7) (которая является отрицательно ориентируемой), а в качестве системы (Б) - систему (2.8) (которая также отрицательно ориентируема). Возникает вопрос о размерах области $D$, фигурирующей в лемме 2.2 .

Предложение 2.5. Система (2.8) при $\sigma n_{0}<2$ в полосе $\left\{(x, y) \in \mathbb{R}^{2}:-1<x<1\right\}$ обладает первым интегралом, который одновременно является и трансцендентной функцией в полосе, и мероморфной функиией во множестве $\left\{(x, y) \in \mathbb{R}^{2}:-1<x<1\right\}$ без начала координат. Последняя точка - единственная существенно особая точка для данного первого интеграла.

Доказательство. После замены переменных

$$
\frac{\sqrt{3}}{2} n_{0} x-y=u, \quad \frac{\sqrt{3}}{2} n_{0} x+y=v
$$

система (2.8) приведется к уравнению

$$
d u\left[u\left(\frac{\sigma n_{0}}{2 \sqrt{3}}+\frac{7}{12}\right)-\frac{v}{12}-\frac{\sigma}{\sqrt{3} n_{0}} u v^{2}\right]+d v\left[\frac{u}{12}+v\left(-\frac{\sigma n_{0}}{2 \sqrt{3}}+\frac{7}{12}\right)+\frac{\sigma}{\sqrt{3} n_{0}} u^{2} v\right]=0 .
$$

После же замены $u=t v, v^{2}=p, v \neq 0$, уравнение примет вид

$$
d p\left[C_{1} t^{2}+C_{2}\right]+2 p\left[C_{1} t-1-u \sqrt{3} \frac{\sigma}{n_{0}} t p\right] d t=0 .
$$

Здесь введены обозначения

$$
C_{1}=7+2 \sqrt{3} \sigma n_{0}, \quad C_{2}=7-2 \sqrt{3} \sigma n_{0} .
$$

Заменой $p=q^{-1}$ приводим последнее уравнение в полных дифференциалах к линейному неоднородному уравнению вида

$$
\frac{d q}{d t}\left[C_{1} t^{2}+C_{2}\right]+2\left[1-C_{1} t\right] q+8 \sqrt{3} \frac{\sigma}{n_{0}} t=0 .
$$

Общее решение однородного уравнения имеет следующий вид:

$$
q_{0}(t)=C \frac{C_{1} t^{2}+C_{2}}{\exp \left\{\frac{2}{C_{1}} \operatorname{arctg} \sqrt{\frac{C_{2} t}{C_{1}}}\right\}}, \quad C=\text { const } .
$$

Варьируя постоянную $C$, получаем дифференциальное уравнение, позволяющее найти трансцендентное решение уравнения (2.10). Особенности возникают лишь при $u=v=0$, т.е. когда значение $t$ не определено. Но последнее уравнение и задает начало координат на плоскости $\mathbb{R}^{2}\{x, y\}$. 
Следствие 2.1. Для системь (2.8) областью притяљсения является вся полоса $\left\{(x, y) \in \mathbb{R}^{2}\right.$ : $-1<x<1\}$.

Следствие 2.2. У системы (2.7) в полосе $\left\{(x, y) \in \mathbb{R}^{2}:-1<x<1\right\}$ не существует замкнутых характеристик.

Доказательство. Начало координат для системы (2.8) является аттрактором (предложение 2.4). В силу предложения 2.5 , область его притяжения - вся полоса. В силу предложения 2.3 попадаем в условия леммы 2.2 .

Следствие 2.3. При $\sigma n_{0}<2$, а также при выполнении условия $B /\left(m n_{0}\right)<\sigma n_{0} / 2$ у систем вида (1.12) при условиях (1.4), (1.11) в полосе П' не существует простых и сложных предельных ииклов, а также любых замкнутых кривых, составленных из траекторий.

\section{3. О ТРАЕКТОРИЯХ, ИМЕЮЩИХ В КАЧЕСТВЕ ПРЕДЕЛЬНЫХ МНОЖЕСТВ БЕСКОНЕЧНО УДАЛЕННЫЕ ТОЧКИ ПЛОСКОСТИ}

3.1. Примеры из динамики твердого тела, взаимодействующего со средой. В этом разделе будут рассмотрены вопросы существования и единственности траекторий динамических систем (1.1) на плоскости, имеющих в качестве $\alpha$ - и $\omega$-предельных множеств бесконечно удаленные точки. Таким образом, на сферах Римана или Пуанкаре предельными множествами данных траекторий будет северный полюс. Такие траектории уже по определению являются ключевыми, поскольку бесконечно удаленная точка всегда является особой.

Для начала рассмотрим системы вида (1.8) и (1.12).

Лемма 3.1. Рассмотрим систему (1.8) на множестве $\Pi \cap\left\{(\alpha, \omega) \in \mathbb{R}^{2}: \omega>0\right\}$. Тогда для любой достаточно гладкой функиии $F \in \Phi$ существует единственная траектория, уходящая 8 бесконечность (имеющая в качестве $\omega$-предельного множества точку $(+0,+\infty)$ ).

Доказательство. Дополним фазовую плоскость $\mathbb{R}^{2}\{\alpha, \omega\}$ бесконечно удаленной точкой. Получим расширенную фазовую плоскость $\overline{\mathbb{R}^{2}\{\alpha, \omega\}}$. Отобразим область $\Pi \cap\left\{(\alpha, \omega) \in \mathbb{R}^{2}: \omega>0\right\}$ на сферу Римана или Пуанкаре. В окрестности северного полюса сферы существуют новые координаты $(\alpha, y), y=-1 / \omega$, в которые переводятся старые координаты из рассматриваемой области расширенной фазовой плоскости неособым преобразованием.

В переменных $(\alpha, y)$ система (1.8) эквивалентна уравнению

$$
\frac{d \alpha}{d y}=\frac{y+\frac{\sigma}{I} y^{2} F(\alpha) \cos \alpha+\sigma \sin \alpha}{\frac{y^{4}}{I} F(\alpha)-\sigma y \cos \alpha+\frac{\sigma}{I} y^{3} F(\alpha) \sin \alpha} .
$$

При этом траектории данного уравнения параметризованы по-другому, нежели траектории системы (1.8).

Видно, что у уравнения существует особая точка $(0,0)$, соответствующая бесконечно удаленной точке $(0,+\infty)$ системы (1.8). Нетрудно убедиться в том, что точка $(0,0)$ уравнения является гиперболическим седлом, что и доказывает лемму.

Лемма 3.2. Рассмотрим систему (1.12) на множестве $\Pi \cap\left\{(\alpha, \omega) \in \mathbb{R}^{2}: \omega>0\right\}$. Тогда для любых достаточно гладких функиий $F$ и s существует единственная траектория, уходящая 8 бесконечность (имеющая в качестве $\omega$-предельного множества точку $(+0,+\infty)$ ).

Доказательство. Следуя методу доказательства леммы 3.1, отображая расширенную фазовую плоскость на сферу, делая аналогичную замену координат, приходим к уравнению

$$
\frac{d \alpha}{d y}=\frac{y+\frac{\sigma}{I} y^{2} F(\alpha) \cos \alpha+\sigma \sin \alpha+y^{2} \frac{s(\alpha)}{m} \sin \alpha}{\frac{y^{4}}{I} F(\alpha)-\sigma y \cos \alpha+\frac{\sigma}{I} y^{3} F(\alpha) \sin \alpha-y^{3} \frac{s(\alpha)}{m} \cos \alpha} .
$$


При этом траектории данного уравнения параметризованы по-другому, нежели траектории системы (1.12).

Видно, что у уравнения существует особая точка $(0,0)$, соответствующая бесконечно удаленной точке $(0,+\infty)$ системы $(1.12)$. Нетрудно убедиться, что данная особая точка имеет топологический тип грубого седла, что и доказывает лемму.

3.2. Существование и единственность траекторий, уходящих на бесконечность. Рассмотрим произвольную автономную систему дифференциальных уравнений (1.1) на плоскости. Будем сопоставлять данной системе уравнение, фазовые траектории которого параметризованы по-другому, а также последние отображены с расширенной фазовой плоскости системы на сферу Римана (или Пуанкаре). При этом, как уже отмечалось, бесконечно удаленные точки перейдут в северный полюс сферы.

\section{Теорема 3.1.}

(1) Если после замены фазовых переменных $\left(x_{1}, x_{2}\right) \rightarrow\left(x_{1}, y\right)$, где $y=1 / x_{2}$, у уравнения, заданного на сфере, появилась особая точка $\left(x_{1}^{0}, 0\right)$, то у рассматриваемой системы существует траектория, стремящаяся $\kappa$ прямой $\left\{\left(x_{1}, x_{2}\right) \in \mathbb{R}^{2}: x_{1}=x_{1}^{0}\right\}$ и имеющая в качестве предельного множества бесконечно удаленную точку.

(2) Если после замены фазовых переменных $\left(x_{1}, x_{2}\right) \rightarrow\left(y, x_{2}\right)$, где $y=1 / x_{1}$, у уравнения, заданного на сфере, появилась особая точка $\left(0, x_{2}^{0}\right)$, то у рассматриваемой системъ существует траектория, стремящаяся $\kappa$ прямой $\left\{\left(x_{1}, x_{2}\right) \in \mathbb{R}^{2}: x_{2}=x_{2}^{0}\right\}$ и имеющая в качестве предельного множества бесконечно удаленную точку.

Доказательство. Следуя леммам 3.1, 3.2, дополним фазовую плоскость бесконечно удаленной точкой, получив $\overline{\mathbb{R}^{2}\{\alpha, \omega\}}$. Отобразим расширенную плоскость на сферу Римана или Пуанкаре. В окрестности северного полюса сферы можно ввести координаты, отображающие эту окрестность на некоторую окрестность нуля координатной плоскости такие, что в случае (1) они равны $\left(x_{1}, y\right), y=1 / x_{2}$, а в случае $(2)-\left(y, x_{2}\right), y=1 / x_{1}$. В первом случае изучаем бесконечно удаленные точки вдоль оси $x_{2}$, а во втором - вдоль оси $x_{1}$. Дальнейшие рассуждения аналогичны доказательствам лемм 3.1, 3.2.

Замечание 3.1. Количество траекторий, уходящих на бесконечность, определяется через топологический тип бесконечно удаленной особой точки. В частности, в системах (1.8) и (1.12) существует единственная траектория, уходящая на бесконечность, поскольку бесконечно удаленная особая точка является седлом (если, конечно, отображать не плоскость, а фазовый цилиндр).

Замечание 3.2. Могут существовать фазовые траектории, уходящие на бесконечность на фазовой плоскости, вдоль которых обе фазовые переменные неограниченно возрастают. В этом случае после замены $x_{1}=1 / y_{1}, x_{2}=1 / y_{2}$, исследуя топологический тип северного полюса сферы, который всегда является особой точкой, можно будет доказать существование и единственность траекторий, приближающихся к прямым вида $A_{1} x_{1}+A_{2} x_{2}+A_{3}=0$, где $A_{1} A_{2} \neq 0$. Действительно, в этом случае траектория будет стремиться к северному полюсу сферы под определенным углом, что соответствует стремлению траектории на плоскости $\mathbb{R}^{2}\left\{x_{1}, x_{2}\right\}$ к прямой, имеющей ненулевой и конечный угловой коэффициент наклона.

3.3. Элементы теории монотонных векторных полей. Рассмотрим семейство достаточно гладких векторных полей $v_{e}$ в области $D$ двумерной ориентированной римановой поверхности. В касательном пространстве $T_{q} D$ каждой точки $q \in D$ можно измерять углы между векторами рассматриваемого семейства.

Определение 3.1. Однопараметрическое семейство полей $v_{e}(e \in E)$ обладает свойством монотонности $(\mathrm{CM})$ в $D$, если для любых точек $q \in D, e_{1} \in E, e_{2} \in E$ в касательном пространстве $T_{q} D$ угол между векторами $v_{e_{1}}, v_{e_{2}} \in T_{q} D$ является монотонной функцией разности параметров $e_{2}-e_{1}$; при этом сохраняется ориентация изменения угла. Если рассматриваемая монотонная зависимость строгая, то говорим, что $v_{e}$ обладает строгим свойством монотонности (строгим CM). 
Теорема 3.2. Пусть поле $v_{e}$ обладает $C M$ в области $D$ плоскости $\mathbb{R}^{2}$. Пусть $x_{0}-$ неособое начальное условие для фазовой траектории поля $v_{e}$ при всех е $\in$ E.

Если для любого е $\in$ E предельное множество траекторий, начинающихся в $x_{0}$, есть множество $g_{0}$, лежащее в конечной части плоскости, причем $\{A, B\}=\partial g_{0}, A$ - предельное множество траектории поля $v_{e_{1}}$, а $B$ - предельное множество траектории $v_{e_{2}}, e_{1}<e_{2}$, то е $\in\left(e_{1}, e_{2}\right)$ тогда и только тогда, когда существует множество $C$, являющееся предельным множеством траектории поля $v_{e}$, причем при увеличении е предельное множество монотонно смещается от $A$ до В. (Здесь идет речъ одновременно либо об $\alpha$-, либо об $\omega$-предельных множествах семейства траекторий.) При этом искомая фазовая траектория единственна, если СМ строгое.

Схема доказательства. По теореме Бендиксона (см. [1]) предельное множество на плоскости только лишь положение равновесия и простой или сложный предельный цикл. Поэтому, в принципе, достаточно разобрать все три случая (данное исследование для краткости опустим).

Можно считать, что для любого $e$ множество $g_{0}$ состоит из $\omega$-предельных множеств. Согласно теореме о непрерывной зависимости решений от начальных условий и правых частей уравнений, при малом изменении параметра $e$ предельное множество останется в близкой окрестности первоначального (если множество $g_{0}$ односвязно). Если последнее множество многосвязно, то последовательно перебираем каждую из компонент связности. В силу выполнения свойства монотонности, при применении теории систем сравнения, немонотонная зависимость траектории от параметра $e$ исключается.

Пусть система обладает строгим СМ. От противного. Пусть для точки $M \in g_{0}$ существуют хотя бы два параметра $e^{1}, e^{2}$, при которых траектории полей $v_{e^{1}}, v_{e^{2}}$ стремятся к точке $M$. Тогда траектории всех полей $v_{\bar{e}}, \bar{e} \in\left[e^{1}, e^{2}\right]$ стремятся к точке $M$ (в силу выполнения СМ). Поскольку $\mathrm{CM}$ строгое, для любого $d>0$ система с векторным полем $v_{e+d}, e+d \in E$, является системой сравнения для $v_{e}$. Легко понять, что траектория поля $v_{e+d}$, выпущенная из неособого начального условия, никогда не пересечет соответствующую траекторию поля $v_{e}$, выпущенную из того же начального условия. В силу последнего, траектории полей $v_{\kappa_{1}}$ и $v_{\kappa_{2}}$ будут иметь разные предельные множества, причем $e^{1}<\kappa_{1}<\kappa_{2}<e^{2}$. Противоречие.

Условия приведенной теоремы можно несколько ослабить для гладкой римановой двумерной ориентированной поверхности $P^{2} \subset \mathbb{R}^{3}$.

Аналогично может быть доказана качественно другая лемма, которая верна и на любых гладких двумерных ориентированных многообразиях.

Лемма 3.3. Рассмотрим семейство полей $v_{e}(e \in E)$ в области сферы $\mathbb{S}^{2}$ следующего вида. Южный $\mathrm{S}$ и северный $\mathrm{N}$ полюса сферы являются седлами. Пусть данное семейство полей обладает строгим СМ таким образом, что при некотором е $e_{1} \omega$-предельным множеством траектории, выходящей из южного полюса, является южный полюс, а при некотором $e_{2}>e_{1} \omega$ предельным множеством траектории, выходящей из северного полюса, является северный полюс. При этом обе рассмотренные ситуачии - это гомоклинические ситуачии на сфере, когда существует лишь одна точка покоя (кроме $\mathrm{N} u \mathrm{~S})$, которая содержится в области, ограниченной указанными сепаратрисами. Других нетривиальных предельных множеств в этой области сферы нет. Тогда существует такое единственное значение параметра $е=e_{0} \in\left(e_{1}, e_{2}\right)$, что траектория, выходящая из южного (северного) полюса, входит в северный (южсный) полюс (это - гетероклиническая ситуачия на сфере).

Доказательство. Единственностъ. Предположим, что указанным свойством обладают два параметра $\bar{e}, \overline{\bar{e}}$. Тогда, в силу строгого СМ, все параметры из интервала $(\bar{e}, \overline{\bar{e}})$ обладают указанным свойством. Рассуждая как в теореме 3.2 , приходим к противоречию со свойством монотонности.

Существование. Таким образом, существует такое единственное значение параметра $e=e_{0}$, что при $e<e_{0}$ реализуется одна гомоклиническая ситуация на сфере, а при $e>e_{0}$ - другая. Предположим, что при $e=e_{0}$ реализуется одна из гомоклинических ситуаций. Тогда существует такая окрестность $U_{e_{0}}^{d}=\left\{e:\left|e-e_{0}\right|<d\right\}$ значения $e=e_{0}$, что для любого $e \in U_{e_{0}}^{d}$ справедлива одна и та же гомоклиническая ситуация. Противоречие. Лемма полностью доказана. 
Замечание 3.3. Мы получили еще один метод доказательства лемм 3.1 и 3.2 . Действительно, искомые поля удовлетворяют условиям леммы 3.3, поскольку бесконечно удаленная точка проектируется в северный полюс сферы Римана (или Пуанкаре), а точка $(\pi / 2,0)$ - в южный.

\section{4. МНОГОМЕРНЫЕ ТОПОГРАФИЧЕСКИЕ СИСТЕМЫ ПУАНКАРЕ И СИСТЕМЫ СРАВНЕНИЯ}

4.1. Топографические системы в многомерном случае. Многомерные ТСП можно определить аналогично ТСП на плоскости. При этом (невырожденные) гиперповерхности уровня коразмерности 1 в пространстве $\mathbb{R}^{n}$ образуют топографическую систему вложенных друг в друга гиперповерхностей, имеющих вершину в особой точке.

Теорема 4.1. Пусть в односвязной области $D$ пространства $\mathbb{R}^{n}$, содержсащеи единственную точку покоя $x_{0}$ достаточно гладкого векторного поля $v_{1}$, существует гиперповерхность $\Gamma \ni x_{0}$, продолжающаяся до гранищь $\partial D$ и пересекающая ее по поверхности $g$ (поверхность $g$ может быть бесконечно удалена), такая, что существует ТСП с иентром в $x_{0}$, задаваемая гладкой функиией $V$, продолюающаяся вдоль $\Gamma$ до $g$, заполняющая область $\mathrm{K} \subseteq D$ и обладаююая свойством

$$
\left.\left(v_{1}, v_{2}\right)\right|_{\mathbb{R}^{n}}>0
$$

$\left(v_{2}=\operatorname{grad} V\right)$ почти всюду в $\mathrm{K}$, за исключением, быть может, некоторых гиперповерхностей, не содержсащих внутри себя $x_{0}$. (Здесь $V=$ const - семейство гиперповерхностей ТСП.) Тогда во всей области D не существует ни одной замкнутой кривой, состоящей из траекторий поля $v_{1}$, пересекающей гиперповерхность Г.

Доказательство. Предположим, что такая кривая $g_{0}$ существует, $\left\{N_{1}, N_{2}\right\}=\Gamma \cap g_{0} \neq \emptyset$ и точка $N_{1}$ - неособое начальное условие при движении по кривой $g_{0}$. Через точку $N_{1}$ проходит замкнутая гиперповерхность $\bar{\Gamma}$ из ТСП, причем $\bar{\Gamma} \subset \mathrm{K}$. Если гиперповерхность $\bar{\Gamma}$ ограничивает область $\bar{S}$, то существует такое $e>0$ (которое уменьшим насколько нужно), что

(1) $N_{e} \in \bar{\Gamma}_{e} \cap \Gamma$, где $\bar{\Gamma}_{e}$ - гиперповерхность ТСП;

(2) расстояние между точками $N_{e}$ и $N_{1}$ равно $e$;

(3) $\bar{\Gamma}_{e}$ ограничивает область $\bar{S}_{e} \supset \bar{S}$.

Выбранное значение $e$ таково, что через конечное время точка, двигаясь по траектории $g_{0} \mathrm{c}$ начальным условием $N_{1}$, покинет область $\bar{S}_{e}$. Поскольку $\bar{S}_{e} \subset \mathrm{K}$ и выполнено неравенство теоремы почти всюду в $\mathrm{K}$, за исключением, быть может, некоторых гиперповерхностей, не содержащих внутри себя $x_{0}$, то точка с начальным условием $N_{1}$ никогда больше в область $\bar{S}_{e} \subset \mathrm{K} \subset D$ не вернется. Так как $\bar{S} \subset \bar{S}_{e}$, то приходим к противоречию с замкнутостью кривой $g_{0}$.

Напомним, что многомерные ТСП особенно удачно помогают решить в ряде случаев проблему различения центра и фокуса. В последнем случае вовсе не обязательно иметь ТСП с центром в данной особой точке. Система сравнения может иметь либо притягивающую, либо отталкивающую особую точку.

Характеристическая функция в многомерном случае строится следующим образом. Пусть $v_{1}, v_{2}$ - два гладких векторных поля в пространстве $\mathbb{R}^{n}$. По полю $v_{1}$ строится (неоднозначно) нормальное гладкое векторное поле $n$. В каждом конкретном случае поле $n$ строится из тех соображений, которые позволяют получить в дальнейшем знакоопределенную характеристическую функцию. Последняя определяется как скалярное произведение $\chi=\left(n, v_{2}\right)$.

Лемма 2.2 с некоторыми уточнениями справедлива и в многомерном случае.

4.2. Четномерный случай. В четномерном случае характеристическая функция имеет наиболее естественный вид.

Пример. Рассмотрим систему слабо перевязанных маятников, т.е. консервативную систему с малыми (неконсервативными) добавками, задаваемую полем $\left\{X_{1}, X^{1}, \ldots, X_{n}, X^{n}\right\}$ в координатах $x=\left\{x_{1}, x^{1}, \ldots, x_{n}, x^{n}\right\}$ следующего вида:

$$
X_{i}=-x^{i}+e F_{i}(x), \quad X^{i}=G_{i}(x)+e F^{i}(x), \quad d G(0) \geqslant 0 .
$$


Тогда естественно выбрать характеристическую функцию в виде

$$
\chi=\sum_{i=1}^{n}\left(X_{i} Y^{i}-X^{i} Y_{i}\right)
$$

где векторное поле $Y$ системы сравнения имеет вид $Y=\left\{Y_{1}, Y^{1}, \ldots, Y_{n}, Y^{n}\right\}$, где $Y_{i}=-x^{i}$, $Y^{i}=G_{i}(x)$.

В многомерном случае, подобно двумерным ТСП и системам сравнения, можно также получить соответствующие утверждения.

\section{СПИСОК ЛИТЕРАТУРЫ}

1. Бендиксон И. О кривых, определяемых дифференциальными уравнениями// Усп. мат. наук. - 1941. - 9. - C. 119-211.

2. Брюно А. Д. Локальный метод нелинейного анализа дифференциальных уравнений. - М.: Наука, 1979.

3. Бурбаки Н. Интегрирование. - М.: Наука, 1970.

4. Голубев В. В. Лекции по аналитической теории дифференциальных уравнений. - М.-Л.: Гостехиздат, 1950.

5. Голубев В. В. Лекции по интегрированию уравнений движения тяжелого твердого тела около неподвижной точки. - М.-Л.: Гостехиздат, 1953.

6. Гуревич М. И. Теория струй идеальной жидкости. - М.: Наука, 1979.

7. Дубровин Б. А., Новиков С. П., Фоменко А. Т. Современная геометрия. - М.: Наука, 1979.

8. Иванова Т. А. Об уравнениях Эйлера в моделях теоретической физики// Мат. заметки. $-1992 .-52$, № 2. - C. $43-51$.

9. Козлов В. В. О падении тяжелого твердого тела в идеальной жидкости// Изв. АН СССР. Мех. тв. тела. - 1989. - 5. - С. 10-17.

10. Ламб Г. Гидродинамика. - М.: Физматгиз, 1947.

11. Локшин Б. Я., Привалов В. А., Самсонов В. А. Введение в задачу о движении тела в сопротивляющейся среде. - М.: МГУ, 1986.

12. Локшин Б. Я., Самсонов В. А., Шамолин М. В. Маятниковые системы с динамической симметрией// Совр. мат. прилож. - 2016. - 100. - С. 76-133.

13. Плисс В. А. Интегральные множества периодических систем дифференциальных уравнений. - М.: Наука, 1967.

14. Пуанкаре A. О кривых, определяемых дифференциальными уравнениями. - М.-Л.: ОГИЗ, 1947.

15. Самсонов В. А., Шамолин М. В. К задаче о движении тела в сопротивляющейся среде// Вестн. Моск. ун-та. Сер. 1. Мат. Мех. - 1989. - 3. - С. 51-54.

16. Табачников В. Г. Стационарные характеристики крыльев на малых скоростях во всем диапазоне углов атаки// в кн.: Тр. ЦАГИ.. - М., 1974. - Т. 1621. - С. 18-24.

17. Чаплыгин С. А. О движении тяжелых тел в несжимаемой жидкости// в кн.: Полн. собр. соч. - Л.: Изд-во АН СССР, 1933. - Т. 1. - С. 133-135.

18. Чаплыгин С. А. Избранные труды. - М.: Наука, 1976.

19. Шабат Б. В. Введение в комплексный анализ. - М.: Наука, 1987.

20. Шамолин М. В. Замкнутые траектории различного топологического типа в задаче о движении тела в среде с сопротивлением// Вестн. Моск. ун-та. Сер. 1. Мат. Мех. - 1992. - 2. - С. 52-56.

21. Шамолин М. В. К задаче о движении тела в среде с сопротивлением// Вестн. Моск. ун-та. Сер. 1. Мат. Мех. - 1992. - 1. - С. 52-58.

22. Шамолин М. В. Классификация фазовых портретов в задаче о движении тела в сопротивляющейся среде при наличии линейного демпфирующего момента// Прикл. мат. мех. - 1993. - 57, № 4. C. $40-49$.

23. Шамолин М. В. Применение методов топографических систем Пуанкаре и систем сравнения в некоторых конкретных системах дифференциальных уравнений// Вестн. Моск. ун-та. Сер. 1. Мат. Мех. $-1993 .-2 .-$ C. $66-70$. 
24. Шамолин М. В. Существование и единственность траекторий, имеющих в качестве предельных множеств бесконечно удаленные точки, для динамических систем на плоскости// Вестн. Моск. ун-та. Cер. 1. Мат. Мех. - 1993. - 1. - C. 68-71.

25. Шамолин M. В. Новое двупараметрическое семейство фазовых портретов в задаче о движении тела в среде// Докл. РАН. - 1994. - 337, № 5. - С. 611-614.

26. Шамолин $M$. В. Введение в задачу о торможении тела в сопротивляющейся среде и новое двухпараметрическое семейство фазовых портретов// Вестн. Моск. ун-та. Сер. 1. Мат. Мех. - 1996. - 4. C. $57-69$.

27. Шамолин М. В. Многообразие типов фазовых портретов в динамике твердого тела, взаимодействующего с сопротивляющейся средой// Докл. РАН. - 1996. - 349, № 2. - С. 193-197.

28. Шамолин М. В. Определение относительной грубости и двупараметрическое семейство фазовых портретов в динамике твердого тела// Усп. мат. наук. - 1996. - 51, № 1. - С. 175-176.

29. Шамолин М. В. Пространственные топографические системы Пуанкаре и системы сравнения// Усп. мат. наук. - 1997. - 52, № 3. - С. 177-178.

30. Шамолин M. В. Об интегрируемости в трансцендентных функциях// Усп. мат. наук. - 1998. - 53, № 3. - C. 209-210.

31. Шамолин М. В. О грубости диссипативных систем и относительной грубости и негрубости систем с переменной диссипацией// Усп. мат. наук. - 1999. - 54, № 5. - С. 181-182.

32. Шамолин M. В. Новое семейство фазовых портретов в пространственной динамике твердого тела, взаимодействующего со средой// Докл. РАН. - 2000. - 371, № 4. - С. 480-483.

33. Шамолин М. В. О предельных множествах дифференциальных уравнений около сингулярных особых точек// Усп. мат. наук. - 2000. - 55, № 3. - С. 187-188.

34. Шамолин М. В. Об интегрировании некоторых классов неконсервативных систем// Усп. мат. наук. 2002. - 57, № 1. - С. 169-170.

35. Шамолин М. В. Сопоставление интегрируемых по Якоби случаев плоского и пространственного движения тела в среде при струйном обтекании// Прикл. мат. мех. - 2005. - 69, № 6. - С. 1003-1010.

36. Шамолин $M$. В. Случай полной интегрируемости в динамике на касательном расслоении двумерной сферы// Усп. мат. наук. - 2007. - 62, № 5. - С. 169-170.

37. Шамолин М. В. Динамические системы с переменной диссипацией: подходы, методы, приложения// Фундам. прикл. мат. - 2008. - 14, № 3. - С. 3-237.

38. Шамолин М. В. Об интегрируемости в элементарных функциях некоторых классов динамических систем// Вестн. Моск. ун-та. Сер. 1. Мат. Мех. - 2008. - 3. - С. 43-49.

39. Шамолин М. В. Трехпараметрическое семейство фазовых портретов в динамике твердого тела, взаимодействующего со средой// Докл. РАН. - 2008. - 418, № 1. - С. 46-51.

40. Шамолин M. В. Новые случаи интегрируемости в пространственной динамике твердого тела// Докл. PAH. - 2010. - 431, № 3. - С. 339-343.

41. Шамолин М. В. Случай полной интегрируемости в динамике четырехмерного твердого тела в неконсервативном поле// Усп. мат. наук. - 2010. - 65, № 1. - С. 189-190.

42. Шамолин М. В. Многопараметрическое семейство фазовых портретов в динамике твердого тела, взаимодействующего со средой// Вестн. Моск. ун-та. Сер. 1. Мат. Мех. - 2011. - 3. - С. 24-30.

43. Шамолин M. В. Некоторые вопросы качественной теории в динамике систем с переменной диссипацией// Совр. мат. прилож. - 2012. - 78. - С. 138-147.

44. Шамолин M. В. Новый случай интегрируемости в пространственной динамике твердого тела, взаимодействующего со средой, при учете линейного демпфирования// Докл. РАН. - 2012. - 442, № 4. - C. 479-481.

45. Шамолин M. В. Новый случай интегрируемости уравнений динамики на касательном расслоении к трехмерной сфере// Усп. мат. наук. - 2013. - 68, № 5 (413). - С. 185-186.

46. Якоби К. Лекции по динамике. - М.-Л.: ОНТИ, 1936.

Шамолин Максим Владимирович

Московский государственный университет имени М. В. Ломоносова (МГУ)

E-mail: shamolin@rambler.ru, shamolin@imec.msu.ru 